\title{
NUMERICAL INVARIANTS FOR SEMICONJUGACY OF HOMEOMORPHISMS OF THE CIRCLE
}

\author{
SHIGENORI MATSUMOTO
}

\begin{abstract}
We give numerical invariants for deciding when two actions of a given group on the circle are semiconjugate. We give conditions for vanishing of an invariant called bounded real Euler class. We also determine endomorphisms of the discrete group $\operatorname{PSL}(2, \mathbf{R})$.
\end{abstract}

1. Introduction, Given two actions of a group on the circle $S^{1}$, Ghys [1] has given a criterion in terms of bounded integral Euler class to decide when they are semiconjugate. In this note, we translate it to numerical invariants and give conditions for vanishing of bounded real Euler class.

Let $G$ be the group of all orientation preserving homeomorphisms of $S^{1}$ and let $\bar{G}$ be its universal covering group. Thus $\bar{G}$ consists of homeomorphisms of $\mathbf{R}$ which commute with $T$, the translation by +1 . For an element $f \in G$, its rotation number is denoted by $\rho(f)(\in \mathbf{R} / \mathbf{Z})$, while for $\bar{f} \in \bar{G}$, its rotation number is denoted by $\operatorname{rot}(\bar{f})(\in \mathbf{R})$. For $f, g \in G$, define

$$
\tau(f, g)=\operatorname{rot}(\bar{f} \bar{g})-\operatorname{rot}(\bar{f})-\operatorname{rot}(\bar{g})
$$

using arbitrary covers $\bar{f}, \bar{g} \in \bar{G}$ of $f$ and $g$. Let $\Gamma$ be a group generated by $\left\{\gamma_{i}\right\}$ and let $\phi_{1}, \phi_{2}: \Gamma \rightarrow G$ be homomorphisms.

Our main results are the following:

THEOREM 1.1. $\phi_{1}$ and $\phi_{2}$ are semiconjugate if and only if

(a) $\rho\left(\phi_{1}\left(\gamma_{i}\right)\right)=\rho\left(\phi_{2}\left(\gamma_{i}\right)\right)$ for any $\gamma_{i}$, and

(b) $\tau\left(\phi_{1}(\gamma), \phi_{1}\left(\gamma^{\prime}\right)\right)=\tau\left(\phi_{2}(\gamma), \phi_{2}\left(\gamma^{\prime}\right)\right)$ for any $\gamma, \gamma^{\prime} \in \Gamma$.

The definition of semiconjugacy will be given in $\S 2$. Let $\chi_{\mathbf{R}}$ be the bounded real Eulder class in $H_{b}^{2}(G: \mathbf{R})$, which will also be described in $\S 2$.

THEOREM 1.2. Let $\phi: \Gamma \rightarrow G$, be a homomorphism from a finitely generated group $\Gamma$. Then the following four conditions are equivalent:

(1) $\phi^{*}\left(\chi_{\mathbf{R}}\right)=0$.

(2) $\phi$ is semiconjugate to a homomorphism into the subgroup of $G$ consisting of translations.

(3) Let $\Omega$ be a minimal set of $\phi$. Then $\phi(\gamma)(x)=x(x \in \Omega)$ implies $\left.\phi(\gamma)\right|_{\Omega}=$ Id.

(4) There is a measure $\mu$ on $S^{1}$ such that $\phi(\gamma)_{*} \mu=\mu$ for all $\gamma \in \Gamma$.

A minimal set of $\phi$ is a nonempty closed subset of $S^{1}$ invariant under the action of $\phi$, which is minimal w.r.t. inclusions. In case $\phi$ has a finite orbit, it is a minimal set and the condition (3) is always satisfied. Otherwise there exists a unique minimal

Received by the editors August 19, 1985 and, in revised form, September 4, 1985.

1980 Mathematics Subject Classification. Primary 57S30; Secondary 55N99, 58F99.

Key words and phrases. Semiconjugacy, bounded cohomology, bounded Euler class. 
set of $\phi$ (see Hector-Hirsch [3]). Finally, as an application of results of Ghys [1] and Matsumoto-Morita [4], we determine endomorphisms of $\operatorname{PSL}(2, \mathbf{R})$.

THEOREM 1.3. Any endomorphism of the discrete group $\operatorname{PSL}(2, \mathbf{R})$ is either the trivial map or the conjugation by an element of $\operatorname{PGL}(2, \mathbf{R})$.

2. Prerequisites. First we shall give a definition of semiconjugacy. A map $h: S^{1} \rightarrow S^{1}$ is called a degree one monotone mapping in case there exists a monotone map $\bar{h}: \mathbf{R} \rightarrow \mathbf{R}$ such that $\bar{h} \circ T=T \circ \bar{h}$ and $\bar{h}$ covers $h$. Note that $h$ may not be continuous, nor injective. Two homomorphisms $\phi_{1}, \phi_{2}: \Gamma \rightarrow G$ are said to be semiconjugate if there exists a degree one monotone mapping $h$ such that $h \circ \phi_{1}(\gamma)=\phi_{2}(\gamma) \circ h$ for any $\gamma \in \Gamma$. Semiconjugacy is an equivalence relation [1]. For $\Gamma=\mathbf{Z}$, it is a classical result that $\phi_{1}$ and $\phi_{2}$ are semiconjugate if and only if $\rho\left(\phi_{1}(1)\right)=\rho\left(\phi_{2}(1)\right)$.

Next let us define bounded Euler class. Let $A=\mathbf{R}$ or $\mathbf{Z}$. Recall that an $A$ valued $n$-cochain $u$ of a group $\Gamma$ is a map $u: \Gamma^{n} \rightarrow A$. $u$ is called a bounded cochain if Image $(u)$ is a bounded subset of $A$. Bounded cochains constitute a cochain subcomplex $C_{b}^{n}(\Gamma: A)$. Its cohomology group $H_{b}^{n}(\Gamma: A)$ is the bounded cohomology group of $\Gamma$. For $\Gamma=G$, an important class of $H_{b}^{2}(G: A)$ called Euler class is defined as follows. A cross section $\sigma: G \rightarrow \bar{G}$ is a map (not a homomorphism) such that $p \circ \sigma=\mathrm{Id}$, where $p: \bar{G} \rightarrow G$ is the canonical projection. $\sigma$ is called bounded if $\sigma(\gamma)(0)$ is bounded. Given a bounded cross section $\sigma$, defined a 2-cochain $e_{\sigma}$ by

$$
e_{\sigma}(f, g)=\sigma(f) \sigma(g) \sigma(f g)^{-1},
$$

where the RHS is a power of $T$, which is identified with an integer by a canonical fashion. $e_{\sigma}$ is in fact a bounded integral cocycle and its cohomology class $\chi_{\mathbf{z}} \in$ $H_{b}^{2}(G: \mathbf{Z})$ is independent of the choice of $\sigma$ and is called the bounded integral Euler class. In fact $\chi_{\mathbf{Z}}$ is a geneator of $H_{b}^{2}(G: \mathbf{Z}) \cong \mathbf{Z}$ (compare Matsumoto-Morita [4]). The image of $\chi_{\mathbf{z}}$ by the map $H_{b}^{2}(G: \mathbf{Z}) \rightarrow H_{b}^{2}(G: \mathbf{R})$ is called the bounded real Euler class and is denoted by $\chi_{\mathbf{R}}$.

The following fundamental theorem is due to Ghys [1]:

THEOREM 2.1. Two homomorphisms $\phi_{1}, \phi_{2}: \Gamma \rightarrow G$ are semiconjugate if and only if $\phi_{1}^{*}\left(\chi_{\mathbf{z}}\right)=\phi_{2}^{*}\left(\chi_{\mathbf{z}}\right)$ in $H_{b}^{2}(\Gamma: \mathbf{Z})$.

3. Numerical invariants. There is a short exact sequence of cochain complexes

$$
0 \rightarrow C_{b}^{n}(\Gamma: \mathbf{Z}) \rightarrow C_{b}^{n}(\Gamma: \mathbf{R}) \rightarrow C^{n}(\Gamma: \mathbf{R} / \mathbf{Z}) \rightarrow 0 .
$$

The induced exact sequence of cohomology groups is

$$
H_{b}^{1}(\Gamma: \mathbf{R}) \rightarrow H^{1}(\Gamma: \mathbf{R} / \mathbf{Z}) \stackrel{\delta_{*}}{\rightarrow} H_{b}^{2}(\Gamma: \mathbf{Z}) \stackrel{\iota *}{\rightarrow} H_{b}^{2}(\Gamma: \mathbf{R}) .
$$

We have $H_{b}^{1}(\Gamma: \mathbf{R}) \cong 0$ for an arbitrary group $\Gamma$ (Gromov [2]). It is also known [2] that for an amenable group $\Gamma, H_{b}^{n}(\Gamma: \mathbf{R}) \cong 0(\forall n)$. In particular, we have $H_{b}^{2}(\mathbf{Z}: \mathbf{Z}) \cong \mathbf{R} / \mathbf{Z}$. For a homomorphism $\phi: \mathbf{Z} \rightarrow G$ it is shown in $[\mathbf{1}]$ that $\phi^{*}(\chi \mathbf{z})=$ $\rho(\phi(1))$ via the above isomorphism.

Now let $\left\{\gamma_{i}\right\}$ be the generators of $\Gamma$ and let $\lambda_{i}: \mathbf{Z} \rightarrow \Gamma$ be the homomorphism given by $\lambda_{i}(1)=\gamma_{i}$. The composite of $\lambda_{i}$ with the canonical projection $\Gamma \rightarrow \Gamma /[\Gamma, \Gamma]$ 
is denoted by the same letter $\lambda_{i}$. Consider the following diagram.

$$
\begin{array}{ccccc}
\operatorname{Hom}(\Gamma /[\Gamma, \Gamma]: \mathbf{R} / \mathbf{Z}) & \rightarrow & H^{1}(\Gamma: \mathbf{R} / \mathbf{Z}) & \stackrel{\delta_{*}}{\rightarrow} & H_{b}^{2}(\Gamma: \mathbf{Z}) \\
\left(\oplus_{i} \lambda_{i}\right)^{*} \downarrow & & \downarrow & & \downarrow \lambda_{i}^{*} \\
\operatorname{Hom}\left(\bigoplus_{i} \mathbf{Z}: \mathbf{R} / \mathbf{Z}\right) & \rightarrow & \bigoplus_{i} H^{\mathbf{1}}(\mathbf{Z}: \mathbf{R} / \mathbf{Z}) & \rightarrow & \bigoplus_{i} H_{b}^{2}(\mathbf{Z}: \mathbf{Z})
\end{array}
$$

Because $\bigoplus_{i} \lambda_{i}: \bigoplus_{i} \mathbf{Z} \rightarrow \Gamma /[\Gamma, \Gamma]$ is a surjection, it follows that the rightmost horizontal map $\bigoplus_{i} \lambda_{i}^{*}$ is injective on Image $\left(\delta_{*}\right)$. This shows

LEMMA 3.1. An element $a \in H_{b}^{2}(\Gamma: \mathbf{Z})$ is zero if $\lambda_{i}^{*}(a)=0$ for any $i$ and $\iota *(a)=0$.

For homomorphisms $\phi_{1}, \phi_{2}: \Gamma \rightarrow G$, we have

LEMMA 3.2. $\phi_{1}$ and $\phi_{2}$ are semiconjugate if and only if

(a) $\rho\left(\phi_{1}\left(\gamma_{i}\right)\right)=\rho\left(\phi_{2}\left(\gamma_{i}\right)\right)$,

(b) $\phi_{1}^{*}\left(\chi_{\mathbf{R}}\right)=\phi_{2}^{*}\left(\chi_{\mathbf{R}}\right)$.

COROLlARY 3.3. For an amenable group $\Gamma, \phi_{1}$ and $\phi_{2}$ are semiconjugate if and only if $\rho\left(\phi_{1}\left(\gamma_{i}\right)\right)=\rho\left(\phi_{2}\left(\gamma_{i}\right)\right)$.

Now let us interpret (b) of Lemma 3.2 using what is to be called the canonical Euler cycle. First we need a concept of $l^{1}$ chains. An $l^{1} n$-chain of $\Gamma$ is an infinite formal sum $c=\sum_{i=1}^{\infty} a_{i}\left(\gamma_{1}^{i}, \ldots, \gamma_{n}^{i}\right)$ such that $\|c\|=\sum_{i=1}^{\infty}\left|a_{i}\right|<\infty$, where $a_{i} \in$ $\mathbf{R}$ and $\gamma_{k}^{i} \in \Gamma$. They form a Banach space $C_{n}^{l^{1}}=C_{n}^{l^{1}}(\Gamma: \mathbf{R})$. The boundary homomorphism $\partial: C_{n}^{l^{1}} \rightarrow C_{n-1}^{l^{1}}$ is defined in a usual manner. It is a bounded operator of Banach spaces. Note that $C_{b}^{n}(\Gamma: \mathbf{R})$ is the dual Banach space of $C_{n}^{l^{1}}$ and the coboundary homomorphism $\delta$ is the dual operator of $\partial \cdot \operatorname{Ker}(\partial)$ and Image $(\partial)$ are denoted by $Z_{n}^{l^{1}}$ and $B_{n}^{l^{1}}$.

LEMMA 3.4. A 2-cocycle $u \in C_{b}^{2}(\Gamma: \mathbf{R})$ is a coboundary if and only if $\left.u\right|_{Z_{2}^{l^{1}}}=0$.

PROOF. The only if part is clear. Suppose $\left.u\right|_{Z_{2}^{l^{1}}}=0$. There is defined a map $v: C_{1}^{l^{1}} \rightarrow \mathbf{R}$ such that $v \circ \partial=u$. To show $v$ is a bounded cochain, i.e., a bounded operator, define $\alpha: C_{1}^{l^{1}} \rightarrow C_{2}^{l^{1}}$ by

$$
\alpha(\gamma)=\sum_{i=1}^{\infty} 2^{-i}\left(\gamma^{2^{i-1}}, \gamma^{2^{i-1}}\right)
$$

$\alpha$ is a bounded operator such that $\partial \circ \alpha=\mathrm{Id}$. For $c \in C_{1}^{l^{1}}$ we have

$$
\|v(c)\|=\|v \circ \partial \circ \alpha(c)\|=\|u \circ \alpha(c)\| \leq\|u\|\|\alpha\|\|c\|,
$$

showing $v$ is bounded. Q.E.D.

REMARK 3.5. Whether the statement of Lemma 3.4 holds for dimension $>2$ or not is an open problem. Compare [4] for related topics.

Define

$$
\beta=\mathrm{Id}-\alpha \circ \partial: C_{2}^{l^{1}} \rightarrow Z_{2}^{l^{1}} \text {. }
$$

Note that $\left.\beta\right|_{Z_{2}^{1}}=$ Id. Thus we have the following corollary. 
COROLLARY 3.6. Let $u$ be a bounded real 2-cocycle. Then we have

(1) $[u]=[u \circ \beta]$ in $H_{b}^{2}(\Gamma: \mathbf{R})$,

(2) $[u]=0$ if and only if $u \circ \beta=0$.

Now we shall prove Theorem 1.1 by applying Corollary 3.6 to $u=e_{\sigma}$, the Euler cocycle. By virtue of Lemma 3.2, it suffices to show the following:

Proposition 3.7. For $\phi: \Gamma \rightarrow G$,

$$
\phi^{*} e_{\sigma} \circ \beta\left(\gamma, \gamma^{\prime}\right)=\tau(f, g),
$$

where $f=\phi(\gamma)$ and $g=\phi\left(\gamma^{\prime}\right)$.

REMARK 3.8. The cocycle $\tau$ is the canonical Euler cocycle. Equality or nullity of bounded real Euler class can be detected by that of the cocycle $\tau$.

PROOF. Naturality of $\beta$ implies

$$
\phi^{*} e_{\sigma} \circ \beta\left(\gamma, \gamma^{\prime}\right)=e_{\sigma} \circ \beta(f, g)=e_{\sigma}(f, g)-e_{\sigma} \alpha(f)-e_{\sigma} \alpha(g)+e_{\sigma} \alpha(f g) .
$$

Let us compute

$$
e_{\sigma} \alpha(f)=e_{\sigma}\left(\sum_{i=1}^{\infty} 2^{-i}\left(f^{2^{i-1}}, f^{2^{i-1}}\right)\right) .
$$

Note that $\sigma\left(f^{k}\right)$ and $\sigma\left(f^{j}\right)$ commute. Identifying the translation $T^{m}$ with the integer $m$, we have

$$
\begin{aligned}
e_{\sigma}\left(\sum_{i=1}^{n} 2^{-i}\left(f^{2^{i-1}}, f^{2^{i-1}}\right)\right) & =2^{-n} \prod_{i=1}^{n}\left(\sigma\left(f^{2^{i-1}}\right)^{2} \sigma\left(f^{2^{i}}\right)^{-1}\right)^{2} \\
& =2^{-n} \sigma(f)^{2^{n}} \sigma\left(f^{2^{n}}\right)^{-1} \rightarrow \operatorname{rot} \sigma(f) \text { as } n \rightarrow \infty
\end{aligned}
$$

Thus

$$
e_{\sigma} \beta(f, g)=\sigma(f) \sigma(g) \sigma(f g)^{-1}-\operatorname{rot} \sigma(f)-\operatorname{rot} \sigma(g)+\operatorname{rot} \sigma(g f) .
$$

The RHS is independent of the choice of the lifts $\sigma(f), \sigma(g)$ and $\sigma(f g)$ of $f, g$ and $f g$. In particular, one can take $\sigma(f)=\bar{f}, \sigma(g)=\bar{g}$ and $\sigma(f g)=\bar{f} \bar{g}$. This shows Proposition 3.7. Q.E.D.

4. Proof of Theorem 1.2. Let $\Gamma$ be a group generated by a finite set $\left\{\gamma_{i}\right\}$ and let $\phi: \Gamma \rightarrow G$ be a homomorphism.

$(1) \Rightarrow(2)$. Vanishing of the real Euler class $\phi^{*}\left(\chi_{\mathbf{R}}\right)$ implies, via Corollary 3.6 and Proposition 3.7, that $\tau\left(\phi(\gamma), \phi\left(\gamma^{\prime}\right)\right)=0\left(\forall \gamma, \gamma^{\prime} \in \Gamma\right)$. Thus $\rho \circ \phi: \Gamma \rightarrow \mathbf{R} / \mathbf{Z}$ is a homomorphism. Let $r: \mathbf{R} / \mathbf{Z} \rightarrow G$ be the standard embedding onto the subgroup of translations. Then $\phi$ is semiconjugate to $r \circ \rho \circ \phi$, by Theorem 1.1.

$(2) \Rightarrow(1)$. Clear.

$(2) \Rightarrow(3)$. First consider the case where each $\rho\left(\phi\left(\gamma_{i}\right)\right)$ is rational. Then $\phi$ has finite orbits, because this property is unchanged by semiconjugacy. This implies (3).

Next consider the opposite case. Let $h, h^{\prime}$ be degree one monotone maps such that $h \circ \phi(\gamma)=r \rho \phi(\gamma) \circ h$ and $\phi(\gamma) \circ h^{\prime}=h^{\prime} \circ r \rho \phi(\gamma)$. Let $\Omega$ be the minimal set of $\Omega$. Suppose $\phi(\gamma) x=x(X \in \Omega)$. Then $h(x)$ is a fixed point of $r \rho \phi(\gamma)$. Thus $r \rho \phi(\gamma)=$ Id. Therefore, $\phi(\gamma)$ keeps the points of $\Omega=\overline{\operatorname{Image}\left(h^{\prime}\right)}$ fixed.

$(3) \Rightarrow(2)$. Proof are divided into three cases. 
Case 1. $\phi$ has a finite orbit. It is easy to deduce (2).

Case 2. The minimal set $\Omega$ is the whole of $S^{1}$. First we shall show the following

ClAIM. Some of $\rho\left(\phi\left(\gamma_{i}\right)\right)$ is irrational.

PROOF. If not, (3) implies that some iterate of $\phi\left(\gamma_{i}\right)$ is the identity. Passing to a conjugation by a homeomorphism of $S^{1}$, we may assume that $\phi\left(\gamma_{i}\right)$ is a translation. Then clearly we have $\phi\left(\gamma_{j}\right) \phi\left(\gamma_{i}\right)(x)=\phi\left(\gamma_{i}\right) \phi\left(\gamma_{j}\right)(x)$ for some $x \in S^{\mathbf{1}}$. Again by (3), $\phi\left(\gamma_{j}\right) \phi\left(\gamma_{i}\right)=\phi\left(\gamma_{i}\right) \phi\left(\gamma_{j}\right)$. Thus Image $(\phi)$ is an abelian subgroup of $G$. By Corollary 3.3, this implies (2) and thus there exists a finite orbit. A contradiction. Q.E.D.

Assume $\rho\left(\phi\left(\gamma_{i}\right)\right) \notin \mathbf{Q} / \mathbf{Z}$. Suppose that the minimal set $X$ of $\phi\left(\gamma_{i}\right)$ is a Cantor set. Let $(a, b)$ be a component of $S^{1}-X$. Consider the graphs of all iterates of $\phi\left(\gamma_{i}\right)$. Then their intersections with the line $x=b$ tends to $b$ from above while they never hit the segment $a \times(a, b)$. Notice that the condition (3), combined with the assumption $\Omega=S^{1}$, implies that if the graph of a certain $\phi(\gamma)$ intersects with that of $\phi\left(\gamma_{i}\right)^{k}$, then they are identical. This shows that no graphs of $\phi(\gamma)$ can intersect with $a \times(a, b)$. The orbit of $a$ under $\phi$ is not dense in $S^{1}$. This contradicts $\Omega=S^{1}$.

Thus we have that the minimal set of $\phi\left(\gamma_{j}\right)$ is $S^{1}$. A classical fact shows then $\phi\left(\gamma_{i}\right)$ is conjugate to a translation by an irrational number. Passing to a conjugation, assume that $\phi\left(\gamma_{i}\right)$ is itself a translation. Then (considering the graphs again) one can easily deduce that every $\phi(\gamma)$ is also a translation.

Case $3 . \Omega$ is a Cantor set. By a suitable semiconjugation, we can pass to Case 2. Observe that in so doing the condition (3) is preserved.

$(2) \Rightarrow(4)$. Clear.

(4) $\Rightarrow(2)$. Using the measure $\mu$ of (4), define a degree one monotone map $h$ by $h(x)=\int_{0}^{x} d \mu(x)$. Then by the invariance of $\mu$, we have

$$
h \circ \phi(\gamma)(x)=h(x)+\int_{0}^{\phi(\gamma)(0)} d \mu(x) .
$$

This shows (2).

Finally by what we have argued above, we obtain the following:

COROLLARY 4.1. $\phi^{*}\left(\chi_{\mathbf{z}}\right)$ is a torsion if and only if $\phi$ has a finite orbit.

REMARK 4.2. The equivalence of (2) through (4) is indicated in [3, Chapter X]. But we gave the full proof because it is much simpler.

5. Proof of Theorem 1.3. $\operatorname{PSL}(2, \mathbf{R})=$ PSL is considered to be the group of Möbius transformations having the unit disk of $\mathbf{C}$ invariant. Thus it acts on $S^{1}$ and there is a natural inclusion map $i:$ PSL $\rightarrow G$. Using a result of Sah-Wagoner [5], Matsumoto-Morita [4] showed that $H_{b}^{2}(\mathrm{PSL} ; \mathbf{R})$ injects onto a subgroup of $H^{2}$ (PSL; R) generated by the Euler class. Together with the exact sequence given at the beginning of $\S 3$, this shows that $H_{b}^{2}(\mathrm{PSL} ; \mathbf{Z})$ is a free cyclic group generated by $i^{*}\left(\chi_{\mathbf{z}}\right)$. It is not difficult to show that $\left\|i^{*}\left(\chi_{\mathbf{z}}\right)\right\|=1 / 2$, where $\|\cdot\|$ is the norm of the corresponding bounded real cohomology class. (Compare [1 and 2].) Let $f$ be an arbitrary endomorphism of PSL. Then $\left\|f^{*} i^{*}(\chi \mathbf{z})\right\| \leq 1 / 2$. Therefore there are but three possibilities to be considered:
(a) $f^{*} i^{*}(\chi \mathbf{z})=0$
(b) $f^{*} i^{*}(\chi \mathbf{z})=i^{*}(\chi \mathbf{z})$
(c) $f^{*} i^{*}(\chi \mathbf{z})=-i^{*}(\chi \mathbf{z})$. 
Case (a). By Theorem 1.2, the map if is semiconjugate to the trivial map. This implies that if $(a)$ has a fixed point for any $a \in$ PSL. Thus for $a$ of finite order, $f(a)=1$. Next for a hyperbolic element $b$, we have $b^{-1}=a b a^{-1}$ for some $a$ of order 2. This shows $f(b)=1$. Any element of PSL is a product of hyperbolic elements; we have $f$ in this case is the trivial map.

Case (b). if is semiconjugate to $i$. Especially $\rho($ if $(a))=\rho(i(a))$. This shows the minimal set of if, as well as that of $i$, is $S^{1}$. Then the degree one monotone map $h$ giving the semiconjugacy must be a homeomorphism (see [1]). Take $a \in$ PSL such that $i(a)$ is a rotation by an irrational number. As is well known, $\rho($ if $(a))=\rho(i(a))$ implies the existence of $b \in$ PSL such that $f(a)=b a b^{-1}$. Now $h i(b)^{-1}$ commutes with $i(a)$, hence with any iterate of it. This shows $h i(b)^{-1}$ is a translation. Therefore $f$ of Case (b) is an inner automorphism.

Case (c). An example of such $f$ is the conjugation $f_{0}$ by $\left[\begin{array}{cc}1 & 0 \\ 0 & -1\end{array}\right]$. Because $f f_{0}^{-1}$ satisfies (b), $f$ of Case (c) is the conjugation by an element of $\operatorname{PGL}(2, \mathbf{R})=$ PSL. Q.E.D.

With a little more work, we also have the following:

COROLlaRY 5.1. Any endomorphism of $\mathrm{SL}(2, \mathbf{R})$ is either the trivial map or the conjugation by an element of $\mathrm{GL}(2, \mathbf{R})$.

We also have the following theorem, whose proof is much easier than Theorem 1.3 .

THEOREM 5.2. Any homomorphism of PSL into $G$ is either trivial or the conjugation by some (maybe orientation reversing) homeomorphism of $S^{1}$, of the natural inclusion $i$.

In [4], it was also shown that $H_{b}^{2}(G ; \mathbf{R}) \cong \mathbf{R}$. This means $H_{b}^{2}(G: \mathbf{Z}) \cong \mathbf{Z}$. This has the following implication. Its proof, using $G$ is simple, is easy and omitted.

THEOREM 5.3. Any endomorphism of $G$ is either trivial or the conjugation by a (possibly orientation reversing) homeomorphism of $S^{1}$.

REMARK 5.4. Ghys has pointed out to the author that Theorem 1.3 has an elementry proof. He has also shown that the same thing is true for $\operatorname{SL}(n, \mathbf{R})$. On the other hand, Tsuboi has shown the author an elementary proof of Theorem 5.3, at least for automorphisms.

PROBLEM. Determine endomorphisms of such groups as $\operatorname{Diff}_{+}^{\infty}\left(S^{1}\right)$.

\section{REFERENCES}

1. E. Ghys, Groupes d'homeomorphismes du cercle et cohomologie borneé, preprint, Université des Sciences et Techniques de Lille I.

2. M. Gromov, Volume and bounded cohomology, Inst. Hautes Études Sci. Publ. Math. 56 (1982), 5-100.

3. G. Hector and U. Hirsch, Introduction to the geometry of foliations, Part B, Aspects of Math. (1983).

4. S. Matsumoto and S. Morita, Bounded cohomology of certain groups of homeomorphisms, Proc. Amer. Math. Soc. (to appear).

5. C.-H. Sah and J. B. Wagoner, Second homology of Lie groups made discrete, Comm. Algebra 5 (6) (1977), 611-642.

Department of Mathematics, College of Science and Technology, Nihon UNIVERSITY, KANDA-SURUGADAI, CHIYODA-KU, TOKYO, JAPAN 